\title{
A Novel Serine Protease Inhibitor Acts as an Immunomodulatory Switch while Maintaining Homeostasis
}

\author{
N. Jiang ${ }^{a} \quad$ S. Thangamani ${ }^{a} \quad$ C.F. Chor ${ }^{a} \quad$ S.Y. Wang ${ }^{a} \quad$ I. Winarsih ${ }^{a} \quad$ R.J. Du ${ }^{a}$ \\ $\begin{array}{lll}\text { J. Sivaraman } & \text { B. Ho } & \text { J.L. Ding } \\ & \text { a }\end{array}$ \\ Departments of a Biological Sciences and ${ }^{b}$ Microbiology, National University of Singapore, Singapore
}

\section{Key Words}

Serine protease inhibitor $\cdot$ Serine proteases $\cdot$ Kinetic

profiles $\cdot$ Prophenoloxidase pathway .

Immunomodulation $\cdot$ Homeostasis

\begin{abstract}
Serine protease cascades boost immune responses while maintaining homeostasis. These crucial actions are intricately regulated by cognate serine protease inhibitors. However, the mechanism underlying such a dynamic immunomodulation during acute phase infection remains obscure, particularly where the pathogen's serine protease adds a new challenge to the host. Here, we found that infection of horseshoe crab, Carcinoscorpius rotundicauda, induced reciprocal profiles of CrSPI (serine protease inhibitor) and CrFurin (serine protease) with respect to their transcription and protein activities. Using recombinant $\mathrm{rCrSPI}$, we explored its inhibitory activity against various microbial proteases and found it most efficacious against a model serine protease, subtilisin A. $\mathrm{rCrSPI}$ inhibited subtilisin at $\mathrm{Ki} 10^{-9} \mathrm{M}$ with a molar ratio of 1 rCrSPI: 2 subtilisin. The $\mathrm{rCrSPI}$ also inhibited plasma CrFurin, suppressed subtilisin-mediated activation of prophenoloxidase (PPO) and interacted with complement C3. Taken together, CrSPI acts as a key immunomodulatory 'on-off'
\end{abstract}

switch in a 2-way regulation of serine protease microbial subtilisin and host serine proteases ( $\mathrm{CrFurin}$ and $\mathrm{CrC} 3$ ), thereby controlling immune responses involving the complements and the PPO-mediated antimicrobial activities, while maintaining homeostasis.

Copyright $\odot 2009$ S. Karger AG, Basel

\section{Introduction}

Serine protease cascades in the host form a common theme which promotes innate immune response while maintaining homeostasis during an infection. Important immunological pathways that have evolved to exploit a general organization of serine protease cascades include the coagulation reaction in horseshoe crab blood [1-3], the fibrinogen clotting cascade in mammalian blood [4], Spätzle-mediated Toll receptor activation in Drosophila [5] and the prophenoloxidase (PPO) pathway in other arthropods [6]. In addition to exploiting its own proteases, the host has evolved a countermeasure which apparently takes advantage of the microbial invader's serine protease

N. Jiang and S. Thangamani share first authorship; J. Sivaraman, B. Ho and J.L. Ding are co-senior authors.

\section{KARGER}

Fax +4161306 1234

E-Mail karger@karger.ch

www.karger.com
(C) 2009 S. Karger AG, Basel

$1662-811 X / 09 / 0015-0465 \$ 26.00 / 0$

Accessible online at:

www.karger.com/jin
Dr. Ding Jeak Ling

Department of Biological Sciences, National University of Singapore

14 Science Drive 4

Singapore 117543 (Singapore)

Tel. +65 6516 2776, Fax +65 6779 2486, E-Mail dbsdjl@nus.edu.sg 
to trigger the PPO pathway, resulting in the production of cytotoxic free radicals to kill the pathogen effectively [7]. Some of the significant advantages in the host-pathogen interaction involving the serine protease cascade organization are: (1) to provide a signal amplification to cause a powerful host response to the microbial invasion [8]; (2) to catalyze a rapid response to restrict the infection to the immediate vicinity, and (3) to ensure that there is tight regulation of the immune response to avoid leakage of undesirable harm to the host itself.

Taken together, serine proteases are indispensible for host immune response. However, at the other extreme, serine proteases that act beyond their restricted immune functions can impose serious injury to the host itself [9]. Therefore, efficient regulation of both the microbial proteases and host proteases is crucial in eliminating the pathogens and in maintaining homeostasis. To achieve this fine balance, the hosts have co-evolved specific serine protease inhibitors (SPIs) to modulate, in a timely manner, the serine proteases associated with the infective microbe as well as the endogenous serine proteases from within the host itself $[9,10]$. For instance, the human Lympho-epithelial Kazal-type Inhibitor (LEKTI) is crucial for controlling the epithelial tissue homeostasis via regulation of trypsin, and a mutation in LEKTI leads to Netherton syndrome [11]. In the horseshoe crab (commonly referred to as Limulus), 3 SPIs called Serpins, also known as Limulus intracellular coagulation inhibitors (LICI-1, LICI-2 and LICI-3) were shown to regulate serine proteases involved in the pathogen-induced coagulation cascade [12].

The co-existence of serine proteases and their cognate inhibitors in the host organism therefore presents the basis for the host to achieve an intricate balance between antimicrobial actions and homeostasis. However, the fundamentals of how such a dynamic immune modulation is achieved judiciously remain unclear. This paper attempts to address this enigma by elucidating the temporal and spatial relationship of an SPI and its cognate proteases during host-pathogen interaction at the acute phase of infection. Our recent work on subtractive expressed sequence tags (ESTs) from the horseshoe crab, Carcinoscorpius rotundicauda [2] led to the discovery of a biochemically active Kazal-like SPI, referred to as CrSPI. The members of the Kazal family of SPIs are either single- or multidomain proteins, with each domain constituting 50-60 amino acids. Typically, there are 6 conserved cysteine residues per domain, which form 3 intradomain disulphide bridges, dictating the molecular conformation of each domain [13, 14]. Kazal protease inhibitors have been shown to be active against fungal and bacterial proteases, and this suggests their participation in the innate immune defense elicited during the microbial invasion [15-17]. Detection of Kazal inhibitors in invertebrates such as ascidian [18], crayfish [19], insects [16] and horseshoe crab [2] suggests that the gene is phylogenetically ancient.

Our preliminary study showed that infection of the horseshoe crab induced a reciprocal profile of temporal changes in the CrSPI versus CrFurin. CrFurin is a subtilisin homolog which belongs to the family of furins/ kexins known to be involved in the processing of protein precursors, which include a large number of immune pro-proteins [20-22]. To further understand the functions of the CrSPI, we cloned and expressed the fulllength CrSPI and investigated its biochemical properties. We found that the recombinant CrSPI (rCRSPI) was able to target several cognate proteases. These include a model microbial serine protease, subtilisin A on one hand, and the host's complement component $\mathrm{C} 3$ and CrFurin on the other hand. Our study has provided novel insights on the immunomodulation of the innate immune response, where CrSPI is a potent multitargeting protease inhibitor, acting as an 'on-off' switch to eliminate the pathogens via the complement and PPO pathways while maintaining host homeostasis.

\section{Materials and Methods}

\section{Animals and Bacterial Challenge}

Horseshoe crabs (C. rotundicauda) were collected from the Kranji Estuary, Singapore. Pseudomonas aeruginosa (ATCC 27853 ) was cultured in LB broth by shaking at $220 \mathrm{rpm}$ for $16 \mathrm{~h}$ at $37^{\circ} \mathrm{C}$. The bacterial pellet obtained by centrifugation at $5,000 \mathrm{~g}$ for $5 \mathrm{~min}$ was washed and resupended to $1 \times 10^{7} \mathrm{cfu} / \mathrm{ml}$ in $0.9 \%$ saline. The horseshoe crabs were injected with a sublethal dose of $1 \times 10^{7} \mathrm{cfu} / \mathrm{kg}$ body weight [23]. The hemocytes, hepatopancreas, heart, intestine and stomach were collected at indicated hours post infection (h.p.i.). Three individual specimens were pooled for each time point.

\section{Immunodetection of Native CrSPI}

The native CrSPI in the tissues was detected by Western analysis. To this end, we identified an 18-mer epitope, PSVCTEEYDPVCVEGKIY, in the CrSPI by predicting the MHC class I binding peptides using SVMHC [24] (www.sbc.su.se/ pierre/svmhc/new. cgi). Polyclonal antibody was raised in rabbits using the synthetic peptide, which was conjugated to BSA carrier. Monospecific antibody directed against CrSPI, which recognizes only the 18-mer peptide was prepared using affinity chromatography. For immunoblotting, the cell-free hemolymph depleted of hemocyanin (HMC-free plasma), the hemocyte lysate and the hepatopancreas lysate were separated on $15 \%$ non-reducing Tricine SDS-PAGE 
and electrotransferred from the gel to a PVDF membrane (Millipore). The CrSPI-monospecific antibody was used at 1:2,000, while HRP-conjugated goat anti-rabbit IgG was used at 1:10,000. The positive reactions were detected with SuperSignal ${ }^{\circledR}$ West Pico chemiluminescent substrate (Pierce, Rockford, Ill., USA).

Spatiotemporal Expression of CrSPI and CrFurin Transcripts

To determine the spatial expression profile of CrSPI, multiple tissue Northern analysis was performed using ${ }^{32} \mathrm{P}-\mathrm{HpF} 219$ EST fragment [2] as the probe. The CrFurin spatial expression profile was determined by reverse transcription PCR amplification using gene-specific primers: forward 5'-GCTGGAATTGGAGGTGTTCG-3' and reverse 5'-CTCCGCCCGATCCACTA-3'. To determine the transcriptional kinetics of CrSPI and CrFurin upon $P$. aeruginosa infection, real-time PCR was conducted on the ABI Prism $^{\mathrm{TM}} 7000$ system (Applied Biosystems, Foster City, Calif., USA) using mRNA templates purified with Oligotex-direct mRNA Mini kit (Qiagen, Hilden, Germany) and the Sybr ${ }^{\circledR}$ Green PCR Master Mix (Applied Biosystems). The primers used for CrSPI were: forward 5'-TCTACTGCTCCTTGTCCTCA- $3^{\prime}$ and reverse 5'-ATCGTACTCTTCAGTACAGAC-3'. Primers used for CrFurin amplification were forward $5^{\prime}$-ATGGATGCTGCAGCAA-TGGTA-3' and reverse 5'-CTGGTTGAAGAA-AGTGTGATCA-3'. Each experiment was performed in duplicates and the cDNA coding for ribosomal protein L3 was used as the endogenous control.

\section{Measurement of Plasma CrFurin Activity and Its Inhibition by $\mathrm{CrSPI}$}

CrFurin activity in the plasma was determined using the Furin-specific fluorigenic substrate (pyroglutamic acid-Arg-ThrLys-Arg-AMC; Calbiochem, Gibbstown, N.J., USA) [25]. To this end, $2 \mu \mathrm{g}$ of the substrate was applied to $1,000 \mu \mathrm{g}$ plasma protein and the mixture was incubated for $30 \mathrm{~min}$ at $37^{\circ} \mathrm{C}$. Then the fluorescence intensity was measured at excitation/emission wavelengths of 320/420 nm using a luminescence spectrofluorimeter LS50B (Perkin Elmer, Waltham, Mass., USA). To examine whether CrSPI inhibits CrFurin directly, increasing amounts of rCrSPI $(0-4 \mu \mathrm{g})$ were added to aliquots each containing $1,000 \mu \mathrm{g}$ protein from the 1 h.p.i. plasma, and the remnant CrFurin activity was measured. All protease activity or protease inhibition assays were performed in triplicates.

\section{cDNA Cloning and Computational Analysis of CrSPI}

Total RNA extracted from the hepatopancreas with Trizol ${ }^{\mathrm{TM}}$ reagent (Invitrogen, Carlsbad, Calif., USA) was used to construct a cDNA library with SMART ${ }^{\mathrm{TM}}$ cDNA library construction kit (Clontech, Mountain View, Calif., USA). The EST clone, HpF219, from the hepatopancreas forward library [2] that showed homology to Kazal protease inhibitor was used as a probe to screen the cDNA library for the full length sequence. The GenBank accession number for the full length CrSPI is DQ090491. The deduced amino acid sequence of CrSPI was aligned with other related $\mathrm{Ka}$ zal protease inhibitors using Clustal X (www.embl.de), and shaded with Boxshade (www.ch.embnet.org). The signal peptides were predicted by SignalP 3.0 server [26]. The hypothetical domain structure of the CrSPI was obtained using the Fold Recognition server, Hhpred [27, 28]. The CrSPI model was built using the SCWRL Server at BIC-JCSG $[29,30]$.

Serine Protease Inhibitor in

Immunomodulation
Recombinant Expression and Mass Spectrometric

Identification of CrSPI and Its Constituent Domains

The full length CrSPI and the 2 constituent domains (CrSPID1 and CrSPI-D2) were expressed using GST fusion system (GE Healthcare, Amersham, UK). The cDNAs encoding the target fragments were amplified by PCR using forward and reverse primers that were designed to introduce a $\mathrm{BamH} 1$ site to the $5^{\prime}$ end and an EcoR1 site to the $3^{\prime}$ end. Such PCR fragments were then digested with BamH1 and EcoR1, and ligated into pGEX 4T1 vector, which were previously linearized by compatible restriction enzymes, and transformed into Escherichia coli, BL 21. Optimum expression of GST fusion protein was induced with 0.5 mM IPTG. The fusion products were isolated by passage through a Glutathione Sepharose 4B column (GE Healthcare). The GST fusion products were cleaved by thrombin for $18 \mathrm{~h}$ at room temperature and chromatographed through a Benzamidine Sepharose column for thrombin removal. The flowthrough, which contained the targeted recombinant protein, was collected. The recombinant proteins were identified by SDS-PAGE and MALDITOF mass spectrometry (MS) prior to further functional study.

\section{Protease Inhibition Assay}

In view of investigating the specificity of CrSPI, the representative host serine protease (trypsin) and bacterial serine proteases (e.g. subtilisin A and proteinase K) were tested. The specificity and purity of the proteases used in this study were first verified by measuring their activities with the corresponding substrates purchased from Sigma [succinyl-Ala-Ala-Pro-Phe-p-nitroanilide (Succ-AAPF-pNA), for subtilisin A and proteinase K, and $N \alpha$ benzoyl-DL-arginine $p$-nitroanilide-hydrochloride (BAPNA) for trypsin].

To measure and compare the inhibitory action of CrSPI on these proteases, the azocoll, which is a common substrate for proteases [31], was used to estimate the remnant protease activities. For each protease tested, $300 \mathrm{ng}$ was mixed with increasing amounts (300-1,500 ng) of rCrSPI in a total reaction volume of $200 \mu \mathrm{l}$, prior to applying to $100 \mu \mathrm{l}$ homogenous azocoll suspension. The mixture was incubated at $37^{\circ} \mathrm{C}$ with shaking at $230 \mathrm{rpm}$ for $2 \mathrm{~h}$ and then the $\mathrm{A}_{490 \mathrm{~nm}}$ of the clear supernatants of the reaction mixtures was read using Spectramax 190 (Molecular Devices, Sunnyvale, Calif., USA). Appropriate negative controls without protease, and positive controls without the inhibitor, were assayed simultaneously. The difference in absorbance between the positive and the negative controls was taken as $100 \%$ protease activity. All reactions were performed in triplicates. A decrease in absorbance indicated the inhibitory effect of rCrSPI towards the protease under investigation.

\section{Inhibition Dynamics of rCrSPI and Its Domains}

The inhibition constant, $\mathrm{K}_{\mathrm{i}}$, which indicates the affinity of the inhibitor for the protease was determined from the rate of substrate hydrolysis in the presence and absence of the inhibitor [3234]. The inhibitory characteristics of rCrSPI were best measured using its most susceptible cognate serine protease. Thus, amongst the bacterial serine proteases examined, subtilisin A (from Bacillus licheniformis), which was found to be most strongly inhibited by the rCrSPI, was chosen to aid our further analysis of the inhibition kinetics of CrSPI. In order to determine the CrSPI inhibition dynamics, aliquots of $250 \mathrm{ng}$ of subtilisin A were first mixed with different amounts of rCrSPI followed by the determination 
of the residual subtilisin activity by using Succ-AAPF-pNA as a chromogenic substrate $\left(\mathrm{E}_{405}=8.8 \times 10^{-3} \mathrm{M}^{-1} \mathrm{~cm}^{-1}\right)$. The action of subtilisin was initiated by adding Succ-AAPF-pNA and the $\mathrm{A}_{405 \mathrm{~nm}}$ was measured spectrophotometrically for up to $5 \mathrm{~min}$. The Michaelis constant, $\mathrm{Km}$, of subtilisin A was determined using substrate Succ-AAPF-pNA. The $1 / \mathrm{Ki}^{\mathrm{app}}$ was determined from the gradient of the graph of $\left(\mathrm{V}_{\mathrm{o}} / \mathrm{V}_{\mathrm{i}}-1\right)$ versus $[\mathrm{I}]$ where $\mathrm{V}_{\mathrm{o}}$ and $\mathrm{V}_{\mathrm{i}}$ are the initial velocities in the absence and presence of inhibitor, respectively, and [I] is the inhibitor concentration. Then $\mathrm{K}_{\mathrm{i}}=$ $\mathrm{Ki}^{\mathrm{app}} /\left(1+[\mathrm{S}] / \mathrm{K}_{\mathrm{m}}\right)$. The inhibition dynamics of $\mathrm{rCrSPI}-\mathrm{D} 1$ and rCrSPI-D2 were calculated as that of rCrSPI.

\section{Stoichiometry of Interaction between Subtilisin and rCrSPI}

The stoichiometry of the subtilisin:rCrSPI complex was determined according to the method of Lingaraju and Gowda [35], by incubating subtilisin with rCrSPI at different molar ratios and then measuring the residual subtilisin activity with Succ-AAPFpNA as described above. The size of the subtilisin:rCrSPI complex was determined by nondenaturing SDS-PAGE.

Cloning of CrFurin, a Subtilisin Homologue in the Host

Degenerate primers: 5'-GAYATGAATGTKAWDCCBGCNTGG-3' (forward) and 5'-GCMGAMGCBGASGTSCCMGTRTG$3^{\prime}$ (reverse) were used to amplify a conserved region of CrFurin from the hemocyte cDNA. Thereafter, gene-specific $5^{\prime}$ RACE primer (5'-TCTAGTTCCATGCCTATTTGAG-3') and $3^{\prime}$ RACE primer (5'-CTTTAGCTGCAAC-ATACAGTA- $\left.3^{\prime}\right)$ were designed to obtain full length cDNA using the Smart RACE kit (Clontech). The nucleotide sequence of CrFurin has been assigned with GenBank accession number, DQ395298.

\section{Measurement of Plasma CrSPI Activity - Inhibition of}

Subtilisin Activity

We rationalized that although subtilisin A is apparently better associated with Bacillus subtilis, the subtilisin homologs have been revealed to be virulence factors and they play important roles in the pathogenesis of Pseudomonas infection. These subtilisin homologs include protease IV, an extracellular serine protease with a molecular mass of approximately $26 \mathrm{kDa}$, and AprA, an alkaline subtilisin-like protease [21,36-38]. To reveal a universal/common strategy that the host organism may exploit to combat the invading microbes, it would appear ideal to test the inhibitory characteristics of CrSPI to these Pseudomonas proteases. However, the lack of commercially available enzymes hindered us from conducting such a test. Nevertheless, our preliminary experiments using $P$. aeruginosa cell lysate have demonstrated that rCrSPI significantly inhibits the subtilisin-like proteases from $P$. aeruginosa (online suppl. fig. 4; for supplementary material, see www.karger.com/doi/10.1159/000209224). Furthermore, CrSPI was found to downregulate plasma CrFurin, a homolog of the subtilisin-type protease.

In view of the above rationalization, it was reasonable for us to pursue the characterization of the inhibitory potential of CrSPI towards subtilisin in order to detect the CrSPI activity in the plasma. To this end, $250 \mathrm{ng}$ of subtilisin was incubated with plasma containing $1,000 \mu \mathrm{g}$ total proteins, along with the subtilisin substrate, Succ-AAPF-pNA, at $37^{\circ} \mathrm{C}$ for $5 \mathrm{~min}$, and then the A405 nm was measured. One unit of subtilisin inhibition activity is defined as the amount of inhibitor required to decrease $\mathrm{A}_{405}$ by 0.001 units in the above assay, compared to a blank control containing phos- phate buffered saline (PBS) instead of plasma protein. Since we have shown that rCrSPI particularly inhibits subtilisin with a very high affinity (in the nanomolar range), the observed inhibition of subtilisin by the plasma proteins would directly correlate with the presence of the active CrSPI in the plasma. All reactions were performed in triplicate.

\section{Measurement of PPO Inhibition by rCrSPI}

Microbial protease, subtilisin A, converts the horseshoe crab hemocyanin from a PPO to its active form, phenoloxidase (PO), which produces highly cytotoxic and reactive quinone with antimicrobial activity [7]. The action of CrSPI to inhibit subtilisin-induced PPO activation was measured using purified horseshoe crab hemocyanin and rCrSPI. To this end, $10 \mu \mathrm{g}$ subtilisin was preincubated with different amounts of rCrSPI for $30 \mathrm{~min}$ at $37^{\circ} \mathrm{C}$, prior to being further incubated with $25 \mu \mathrm{g}$ of purified hemocyanin in the activation buffer $(50 \mathrm{mM}$ Tris- $\mathrm{HCl}, \mathrm{pH} 7.4)$ at $20^{\circ} \mathrm{C}$ for $10 \mathrm{~min}$. Immediately after the addition of $5 \mathrm{mM} 4$-methylcatechol, the phenolic substrate, the $\mathrm{A}_{405 \mathrm{~nm}}$ was monitored using a microplate reader (Molecular Devices). Negative control without subtilisin and positive control without rCrSPI were assayed simultaneously. The difference in $\mathrm{A}_{405}$ between the positive and the negative controls was taken as $100 \%$ PPO-activating ability. All reactions were performed in triplicate.

\section{GST Pull Down and Mass Spectrometric Analysis of CrSPI} Interaction Partner

Recombinant CrSPI protein was used to pull down its interaction partner from various tissues. To achieve this, an aliquot of 40 $\mu \mathrm{l}$ rCrSPI-bound Glutathione Sepharose 4 Fast Flow (GE Healthcare, Amersham, UK) beads was incubated overnight by end-toend rotation with $1.5 \mathrm{ml}$ of each of the following samples: plasma, hemocyte lysate, hepatopancreas lysate, muscle lysate, heart lysate and intestine lysate. Then the bound proteins were eluted for $1 \mathrm{~h}$ with $80 \mu \mathrm{l}$ of $40 \mathrm{~mm}$ reduced Glutathione (Sigma-Aldrich, St. Louis, Mo., USA ). The eluted proteins were resolved on SDSPAGE and stained with Coomassie brilliant blue R-250 (Sigma). Protein bands of interest were treated with in-gel trypsin digestion prior to MS-MS analysis. Void Sepharose were included to demonstrate the specificity. Subtilisin, which was earlier established to bind avidly with CrSPI was used as a positive control, while PBS was the negative control. All procedures were performed at $4^{\circ} \mathrm{C}$.

\section{Results}

\section{Infection Induces Reciprocal Profiles of $m R N A$ and} Plasma Protein Activities of CrSPI and CrFurin

Quantitative real-time PCR revealed the dynamic transcription profile of CrSPI at the acute phase (1-6 h.p.i.) of the $P$. aeruginosa infection (fig. la, solid line). The CrSPI transcription was repressed at 1 h.p.i. and surged upwards from 3 h.p.i. onwards to a peak at 6 h.p.i. Interestingly, between 1 and 3 h.p.i., CrFurin exhibited transcription kinetics which were reciprocal to those of CrSPI, with a significant increase in the transcript level 
Fig. 1. Kinetics of the gene transcription and the plasma protein activities of CrSPI and CrFurin upon bacterial challenge. a Real-time PCR analysis was performed for CrSPI (solid line) and CrFurin (dashed line) using mRNAs from hepatopancreas and hemocytes, respectively. b Plasma CrSPI activity (solid line) and plasma CrFurin activity (dashed line).

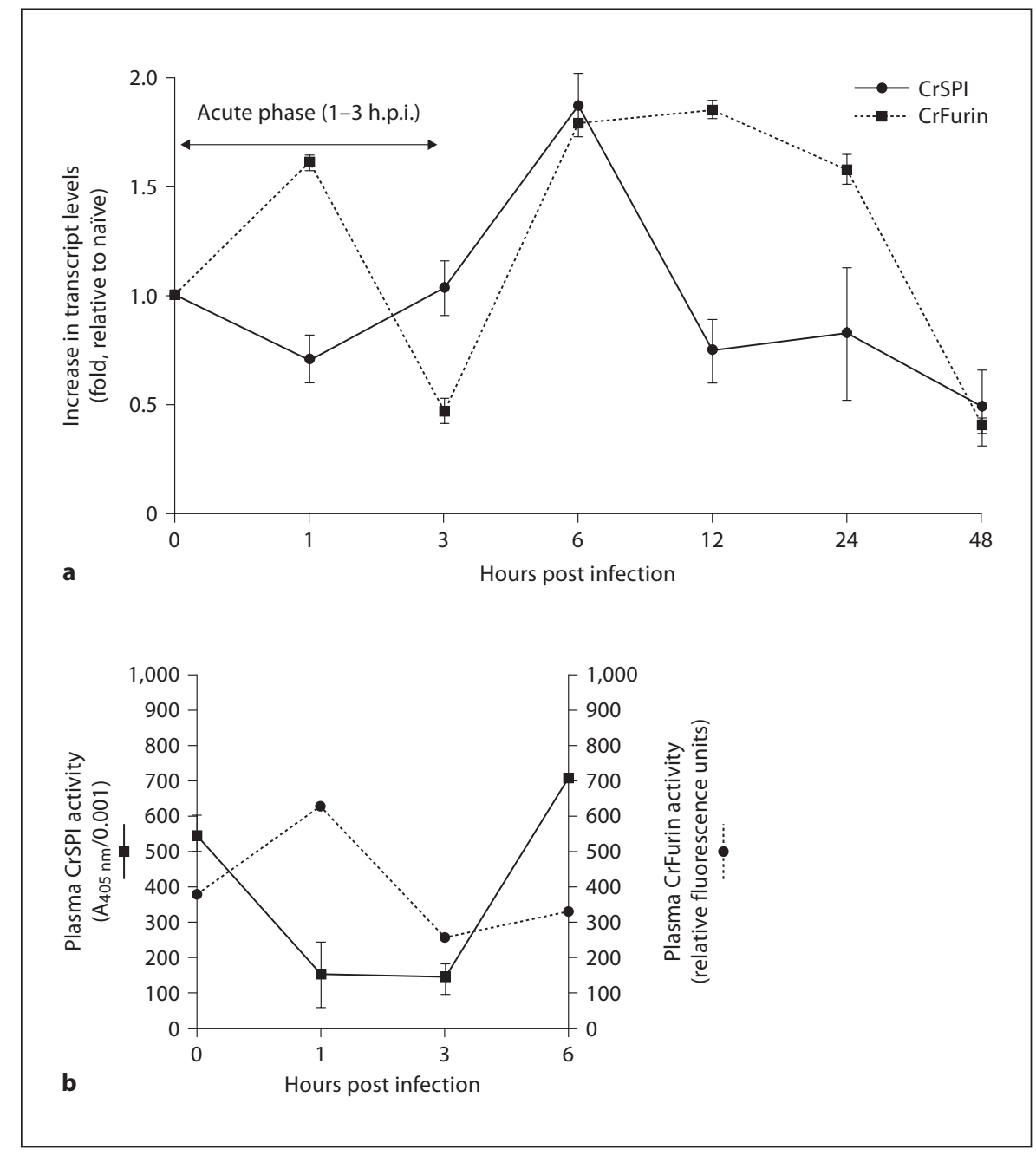

at 1 h.p.i. and a transient repression at 3 h.p.i. (fig. 1a, dashed line).

Plasma samples obtained from various time points of infection were used to investigate the biochemical kinetics of CrSPI and CrFurin. Figure $1 \mathrm{~b}$ shows a reciprocal profile of CrSPI and CrFurin activities in the acute phase plasma, which concurs with the transcriptional kinetics. The functional significance of the reciprocal profile of this protease versus protease inhibitor will be discussed in the following section.

\section{CrSPI Is Exclusively Expressed in the Hepatopancreas}

Northern hybridization showed that CrSPI was expressed exclusively in the hepatopancreas (fig. 2a). In order to trace the native form of CrSPI, we examined samples from various tissues by nonreducing tricine gel followed by Western blot analysis using rabbit anti-CrSPI monospecific antibody. The detection limit of this Western blotting protocol was as low as $10 \mathrm{ng}$ of rCrSPI-D2, according to the $5-\mathrm{kDa}$ band observed in the hemocyanin-free plasma spiked with $10 \mathrm{ng}$ rCrSPI-D2 (fig. 2b, lane 9). With this protocol, we detected a single band of $12 \mathrm{kDa}$ in the hepatopancreas lysate (fig. $2 \mathrm{~b}$ ), which most probably represents the unprocessed 109-mer CrSPI molecule. We also observed a single band of $>100 \mathrm{kDa}$ in both the naïve and infected hemocyte lysate (fig. $2 \mathrm{~b}$, lanes 4-6). Moreover, the band of $>100 \mathrm{kDa}$ was also observed in the hemocyanin-free plasma spiked with $10 \mathrm{ng}$ of the 5-kDa rCrSPI-D2 (fig. 2b, lane 9). However, no band corresponding to mature CrSPI consisting of 84 amino acids with an expected molecular weight of $9.4 \mathrm{kDa}$ was detected in any of the tissues examined (naïve or infected), despite the very high detection sensitivity of the protocol. These observations of the presence of a $>100 \mathrm{kDa}$ band 

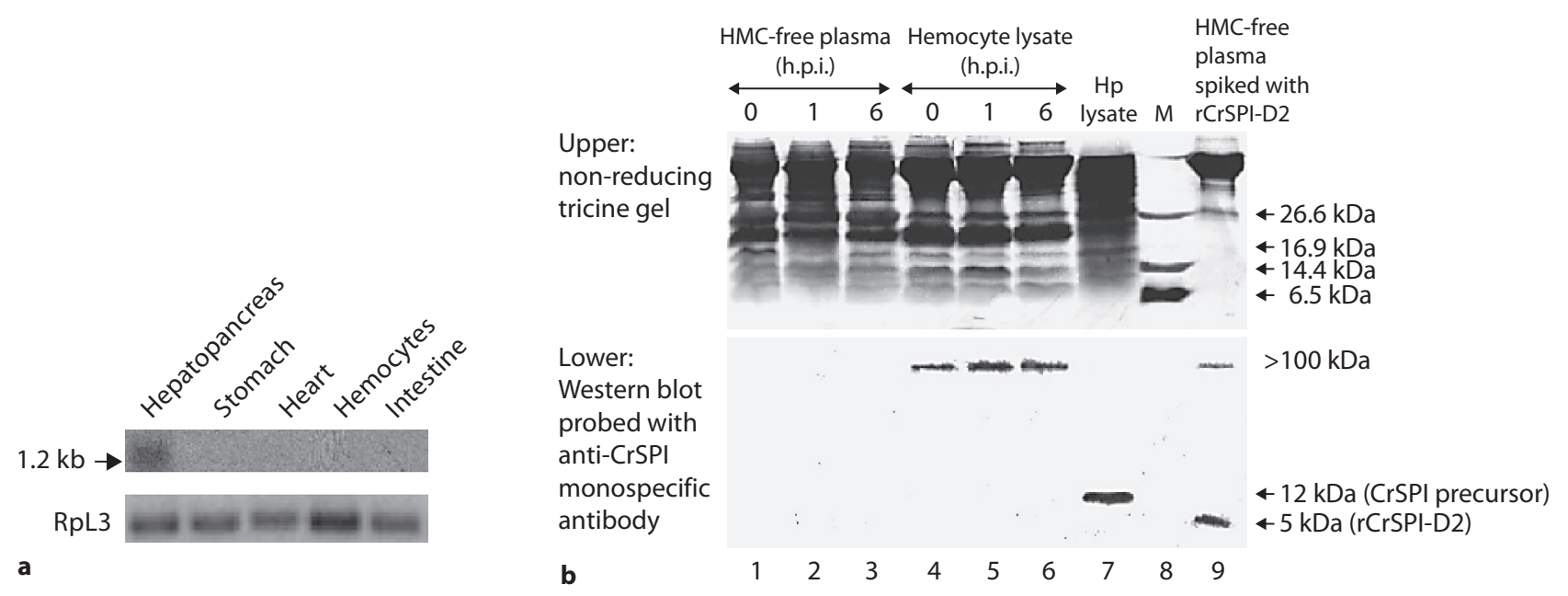

Fig. 2. CrSPI is exclusively synthesized in hepatopancreas. a Multiple tissue Northern analysis of CrSPI was performed using ${ }^{32} \mathrm{P}-\mathrm{HpF} 219$ EST fragment [2] as the probe and ribosomal protein L3 as an endogenous control. b Western blot analysis for the distribution of native CrSPI in various naïve and/or infected tissues.

and yet the absence of the anticipated $9.4 \mathrm{kDa}$ band raised an intriguing question which deserves further consideration and is discussed further in the next section.

\section{Isolation of cDNA Encoding CrSPI and Molecular Characterization of the Protein}

As a member of the family of Kazal protease inhibitors, which has potential to be active against microbial proteases, it was pertinent for us to show the ability of CrSPI to target different serine proteases. Towards this, we used the HpF219 clone from the subtractive hybridization EST [2] as the probe to retrieve a full length Kazal-like SPI from the C. rotundicauda hepatopancreas cDNA library, named as CrSPI. The ORF encodes a protein of 109 amino acids including an N-terminal signal peptide of 25 amino acids (fig. 3a). To reveal the domain structure of CrSPI, we aligned its amino acid sequence with known protease inhibitors [16, 19, 39] (fig. 3b) and illustrated the schematic domain structure in figure $3 \mathrm{c}$. Furthermore, the hypothetical 3D structures of the CrSPI domains were predicted by comparative modeling, as shown in figure $3 \mathrm{~d}$.

As shown in figure 3c, CrSPI contains 2 Kazal-like SPI domains: CrSPI-D1 (domain 1) of 39 amino acids and CrSPI-D2 (domain 2) of 44 amino acids. A closer examination of the primary structure of these domains suggests that CrSPI is unique, deviating from typical Kazal-type SPIs. In general, the conformation of each domain of Kazal- type inhibitor includes 3 rings $-\mathrm{A}, \mathrm{B}$ and $\mathrm{C}$ - formed by the disulfide bridges between the cysteines: $\mathrm{C} 1-\mathrm{C} 5, \mathrm{C} 2-$ C4, C3-C6, respectively [13, 14]. Notably, the second domain, CrSPI-D2, contains all the 6 conserved cysteine residues expected of a typical Kazal SPI domain whereas the first domain, CrSPI-D1, lacks the first and the fifth cysteine residues. Disulfide bond arrangement similar to CrSPI-D1 has been found in GmSPI 2, a single domain

Fig. 3. Isolation of CrSPI, sequence analysis and comparative modeling of domains 1 and 2. a The amino acid sequence of the mature CrSPI. Signal peptide is underlined. The domains, D1 and $\mathrm{D} 2$ are shown in blue and green, respectively. The arrows point to the putative $\mathrm{P} 1$ active sites. $\mathbf{b}$ Multiple alignments of the domains of CrSPI with Kazal protease inhibitors from other organisms. Dip = Dipetalogastin; D1, D2 and D3 = domains 1, 2 and 3, respectively. Database accession numbers: Galleria, aak48526; crayfish, caa56043; Dipetalogastin, caa10384; mouse, np_033284. Conserved cysteines are marked \#, and their intramolecular disulphide bridges are shown. Amino acid residues which are conserved among all organisms are shaded in black. c Schematic representation of the domain organization of CrSPI. The disulphide bond arrangements are indicated. The relative positions of P1 active sites are shown with upward arrows. d Comparative modeling of individual domains of CrSPI isoforms. The modeling for domain 2 was obtained from the first 37 amino acids. The P1 active site residues are in blue. Cys residues at positions 1, 9, 20 and 36 in domain 1 are annotated (magenta) as Cys2, 3, 4 and 6, respectively. Cys residues at positions 2, 6, 14, 24 and 31 in domain 2 are also annotated (magenta) as Cys1, 2, 3, 4 and 5, respectively. The disulphide bridges are shown in yellow. 

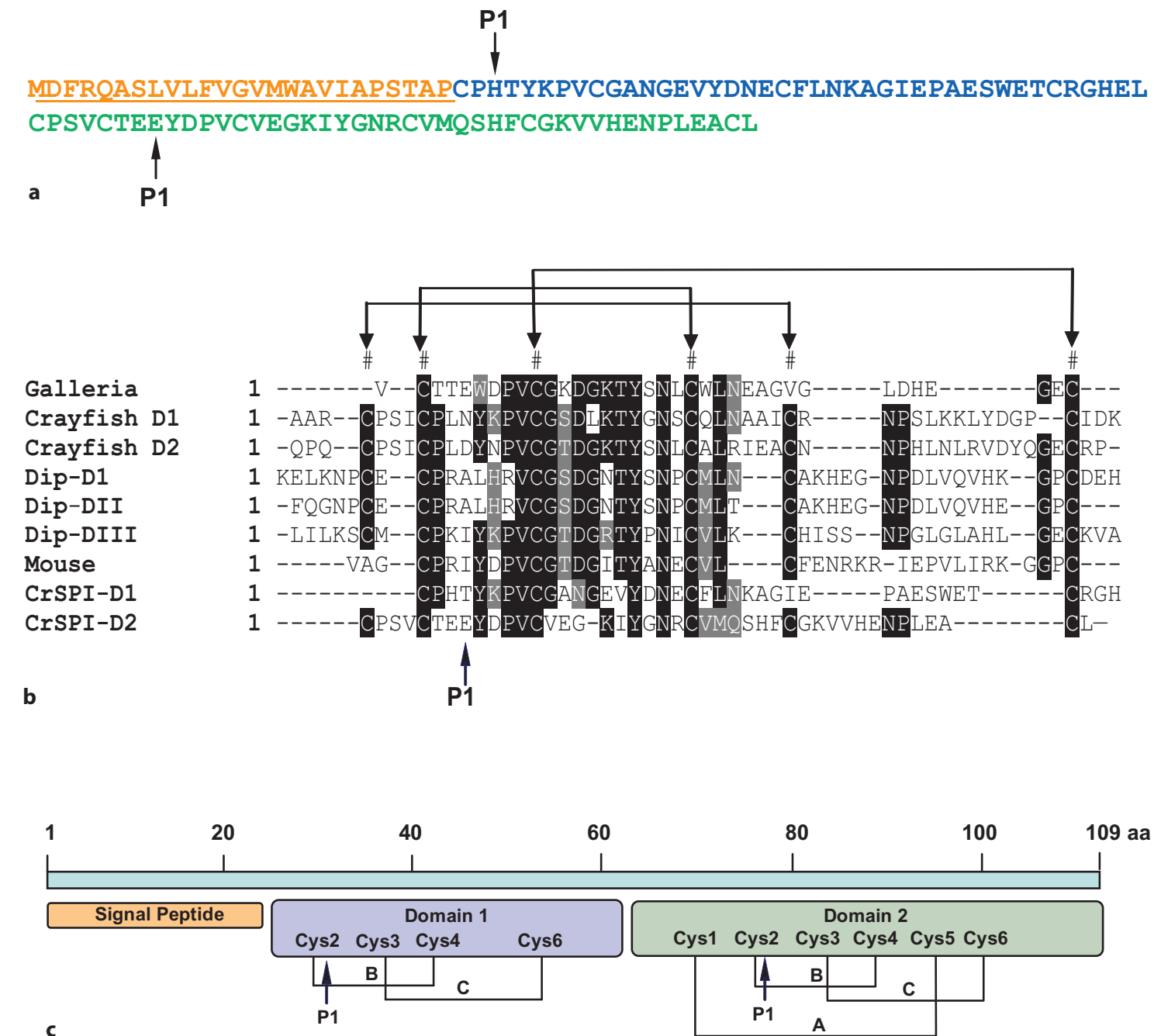

CrSPI-D1

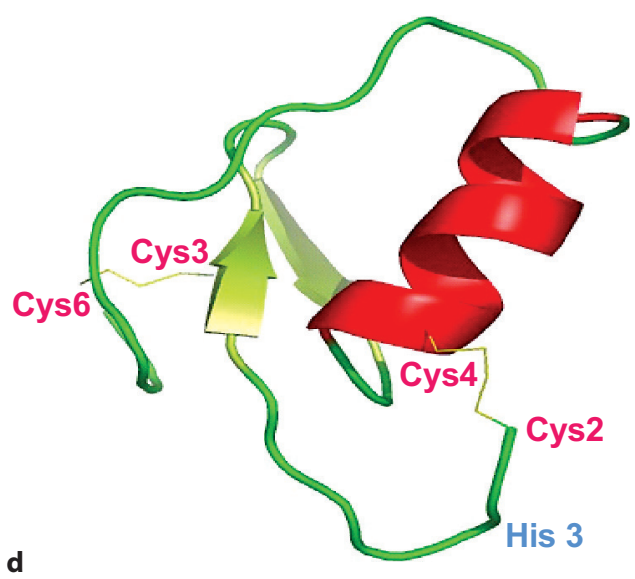

CrSPI-D2

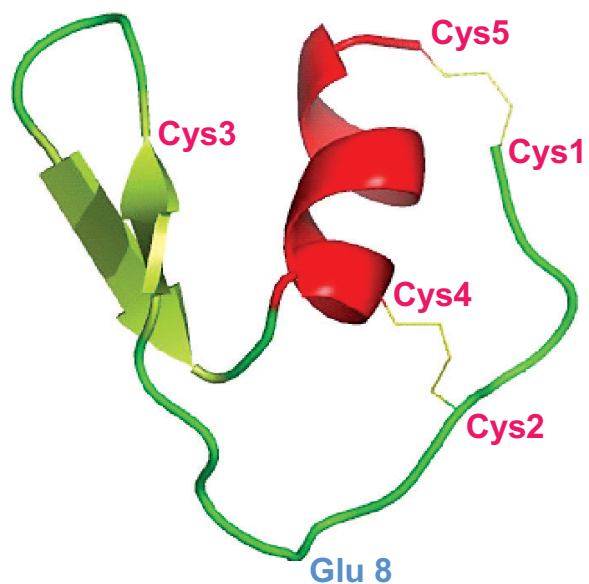




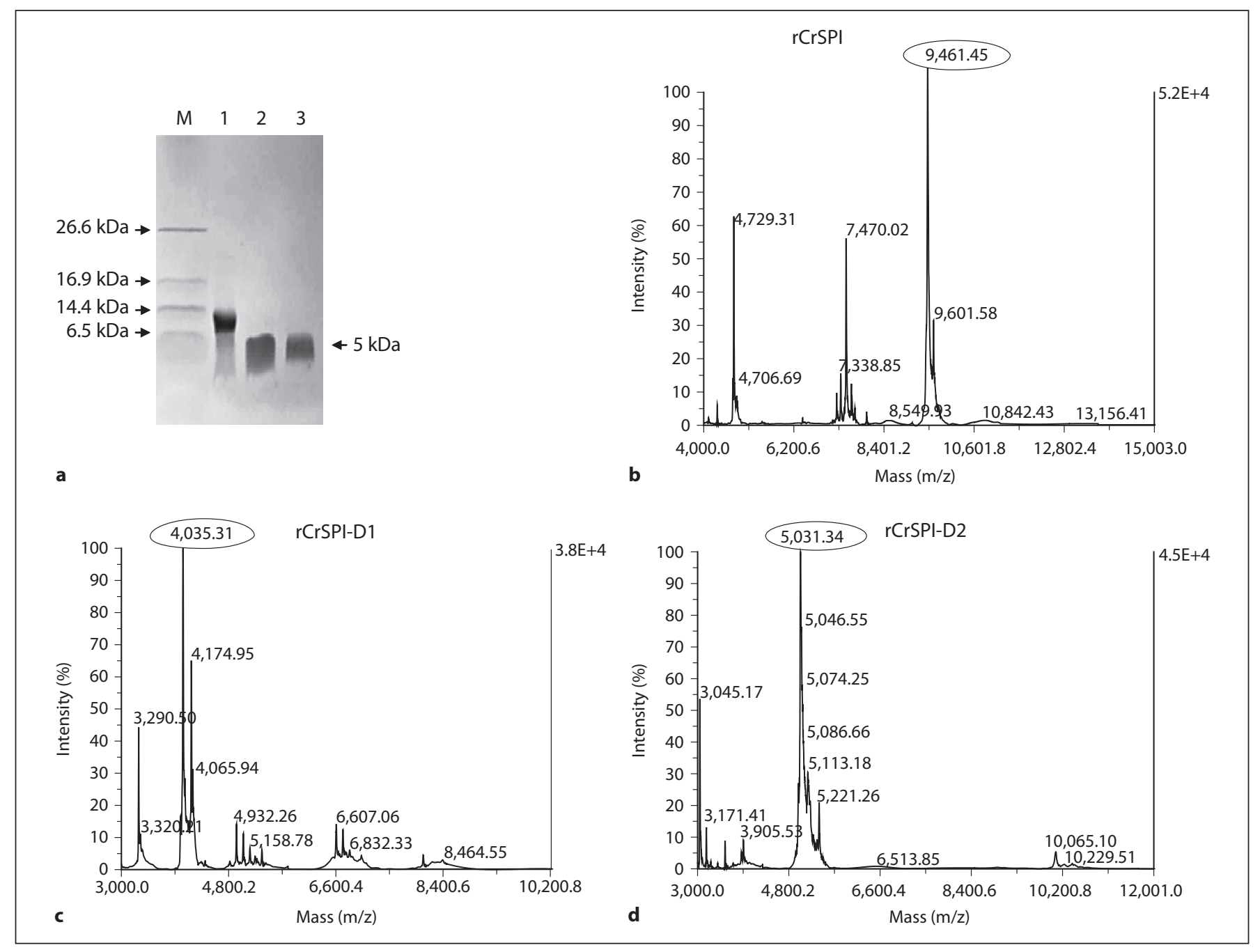

Fig. 4. Mass spectroscopic identification of rCrSPI and its constituent domains. a SDS-PAGE analysis of recombinant proteins under non-reducing condition. $\mathrm{M}=$ Marker; lane $1=\mathrm{rCrSPI}$ (approx. $9 \mathrm{kDa}$ ); lane 2 = rCrSPI-D1 (approx. $4.5 \mathrm{kDa}$ ); lane $3=$

CrSPI-D2 (approx. $5 \mathrm{kDa}$ ). b-d Mass spectra of full length rCrSPI and domains, rCrSPI-D1 and rCrSPI-D2. The molecular weight of each recombinant protein is circled.

Kazal inhibitor isolated from the greater wax moth, Galleria mellonella. GmSPI 2 had been shown to be functionally active in spite of the missing $\mathrm{C} 1-\mathrm{C} 5$ disulfide bond [16]. Therefore, the 2 domains of CrSPI were subcloned and their respective functionalities were further examined.

\section{Preparation of Recombinant CrSPI Protein and Its \\ Individual Domains for Functional Study}

The very low abundance of native CrSPI in the horseshoe crab (fig. 2b), had necessitated us to express rCrSPI and its individual domains for further functional study. To this end, the GST-fusion system was exploited. For

characterizing the rCrSPI function, the GST tag was removed from the purified protein when necessary. The SDS-PAGE profile in figure $4 \mathrm{a}$ shows that significant amounts of pure rCrSPI and its individual domains (CrSPI-D1 and CrSPI-D2) were obtained. The exact molecular weight of each protein was further determined by MS. The spectra in figures $4 \mathrm{~b}-\mathrm{d}$ confirmed the identity of the recombinant proteins procured.

\section{Inhibitory Potentials of $r$ CrSPI towards Its Cognate Proteases}

Previous studies have shown that the P1 site in each domain constitutes the reactive site that determines the 


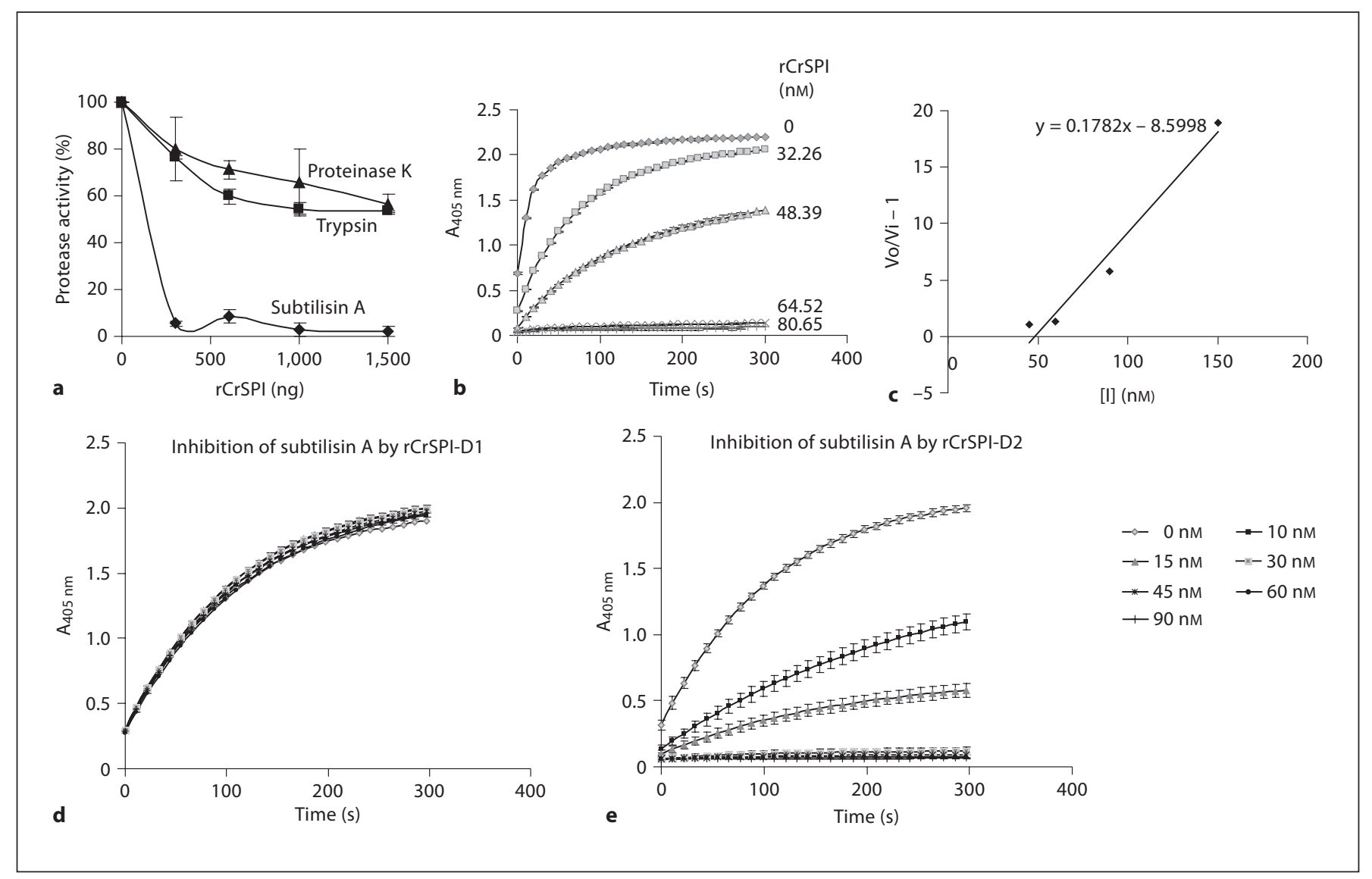

Fig. 5. The inhibitory potential of rCrSPI against its cognate proteases. a Inhibitory specificity of CrSPI. Azocoll assay was performed to determine the inhibitory potential of the rCrSPI against subtilisin A, trypsin and protease K. $\mathbf{b}$ The kinetics of inhibition of subtilisin A by rCrSPI were analyzed spectrophotometrically using subtilisin-specific synthetic substrate. c Determination of $\mathrm{Ki}^{\mathrm{app}}$ for rCrSPI against subtilisin A from the gradient of the

specificity of the Kazal inhibitor [13, 40, 41]. For CrSPI, the $\mathrm{P} 1$ sites in domains 1 and 2 are His and Glu, respectively (fig. 3a). The implication on the inhibitory specificity of such a P1 site profile has not been reported before. To address this, we tested 3 different types of serine proteases: subtilisin A, trypsin and proteinase K. As shown in figure $5 \mathrm{a}$, the rCrSPI showed strongest inhibitory activity against subtilisin A. At $300 \mathrm{ng}$, rCrSPI almost completely inhibited the activity of $250 \mathrm{ng}$ subtilisin A, whereas only $20 \%$ inhibitions were observed with trypsin and proteinase $\mathrm{K}$ under the same condition. The affinity of CrSPI for its cognate protease was characterized by the inhibition constant, $\mathrm{Ki}$, which was determined from the rate of substrate hydrolysis in the presence and absence of the CrSPI (fig. 5b, c) [32-34]. Figures 5d and e show the graph of $(\mathrm{Vo} / \mathrm{Vi}-1)$ vs. [I], where Vo and Vi are the initial velocities in the absence and presence of inhibitor, respectively. [I] = Inhibitor concentration. d, e Similar to $\mathbf{b}$, the kinetics of inhibition of subtilisin A by the 2 domains, rCrSPI-D1 and rCrSPI-D2, were analyzed spectrophotometrically using subtilisin-specific synthetic substrate.

inhibition of subtilisin A by the increasing concentrations of the individual constituent domains, rCrSPI-D1 and rCrSPI-D2, respectively. It is interesting that on its own, CrSPI-D1 showed no inhibitory activity against subtilisin whereas rCrSPI-D2 $(\mathrm{Ki}=0.25 \mathrm{nM})$ showed a 10 fold greater inhibitory capacity compared to full length rCrSPI $(\mathrm{Ki}=2.76 \mathrm{nM})$. Thus, it is tempting to speculate that domain 1 might act as an autoregulator in the intact CrSPI molecule since its absence allowed CrSPI-D2 to exhibit 10-fold higher inhibitory activity. Nevertheless, whether or not CrSPI-D1, which lacks the inhibitory activity by itself, is functional under physiological condition remains to be addressed by future structure-activity relationship studies. 


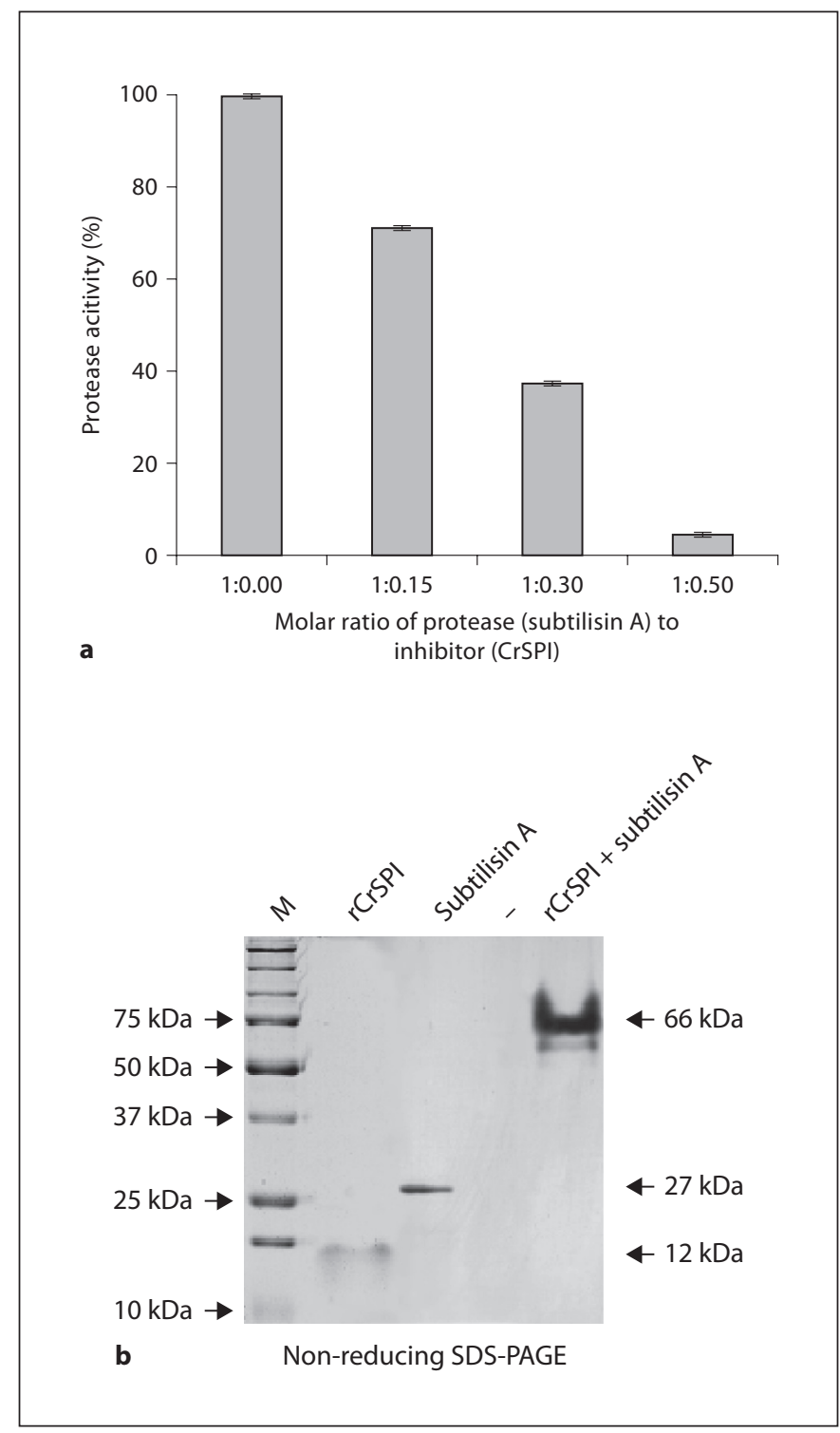

Fig. 6. Stoichiometry of interaction between rCrSPI and subtilisin A. a Various molar ratios of subtilisin A:rCrSPI were incubated and the residual protease activity was measured using chromogenic substrates. b Non-reducing SDS-PAGE analysis of purified rCrSPI and the rCrSPI-subtilisin A complex. The rCrSPI+subtilisin lane shows a band at $66 \mathrm{kDa}$, indicating the complex formation between rCrSPI and subtilisin to be at a stoichiometric ratio of 1:2.

\section{Stoichiometry of Interaction between CrSPI and \\ Subtilisin}

Stoichiometric ratio is an interesting feature of the rCrSPI:subtilisin A complex. The inhibitory kinetic study showed that maximum inhibition was achieved at the ratio of 1 rCrSPI:2 subtilisin A (fig. 6a), suggesting that 1 CrSPI molecule can inhibit 2 subtilisin molecules. The observed stoichiometric ratio was further supported by the nonreducing SDS-PAGE profile of rCrSPI-subtilisin A complex (fig. 6b) and the preliminary crystallographic studies on CrSPI:subtilisin A complex (unpublished data). A similar stoichiometric ratio was previously observed in the crystal structure of the tomato inhibitor:subtilisin [42]. It is also worth mentioning here that 2 other serine protease inhibitors: LEKTI, a 15-domain inhibitor with 2 Kazal-type domains [43], and SPIPm2, a 5-domain Kazal-type inhibitor of the black tiger shrimp [15], most likely engage with more than 1 protease substrate molecule each.

\section{CrFurin: a Potential Endogenous Cognate Serine Protease of CrSPI}

Furin, the eukaryotic homolog of subtilisin, plays an important role in processing proproteins involved in innate immune responses [20-22]. We have cloned the full length cDNA of CrFurin (online suppl. fig. 1) and demonstrated that the CrFurin is exclusively transcribed in the hemocytes (online suppl. fig. 2a). Using furin-specific substrate, we detected CrFurin activity in the plasma (online suppl. fig. 2b). These observations suggest that CrFurin is synthesized in the hemocytes and secreted into the plasma where its function is realized. Almost all mammalian furins have a cytoplasmic tail and a transmembrane domain. Hatsuzawa et al. [44] and Vidricaire et al. [45] have shown that by removing the cytoplasmic tail and transmembrane domain, the furins can be secreted out of the cell as a functional soluble form. Furthermore, over-expression of furin is also known to induce efficient secretion of the truncated form [46]. In some mammals, the ectodomain of furin is shed naturally in the epididymis and the extracellular form may be involved in the fluid and/or sperm membrane protein processing [47].

CrSPI targets subtilisin A at very high affinity, and since CrFurin is a homolog of subtilisin that exists in the host, we sought to address the possibility that CrSPI might target CrFurin as well. The first line of evidence came from the observation that rCrSPI inhibited the CrFurin activity in the plasma in a dose-dependent manner (fig. 7, dashed line), suggesting that CrFurin might be an endogenous cognate serine protease of CrSPI. Whether or not CrSPI interacts directly with CrFurin remains to be investigated.

\section{CrSPI Regulates PPO Activity via Modulation of \\ Microbial Protease}

CrSPI has been shown to inhibit the microbial protease subtilisin A with high affinity; it is also known that 
Fig. 7. $\mathrm{rCrSPI}$ inhibited the subtilisin-mediated PPO activation and the endogenous CrFurin activity in the plasma. The solid line shows the phenoloxidase (PO) activity induced by subtilisin which was preincubated with increasing amounts of rCrSPI. Increasing dose of rCrSPI resulted in progressively lower PO activity. The dashed line indicates the residual CrFurin activity when 1 h.p.i. plasma was incubated with increasing doses of rCrSPI.

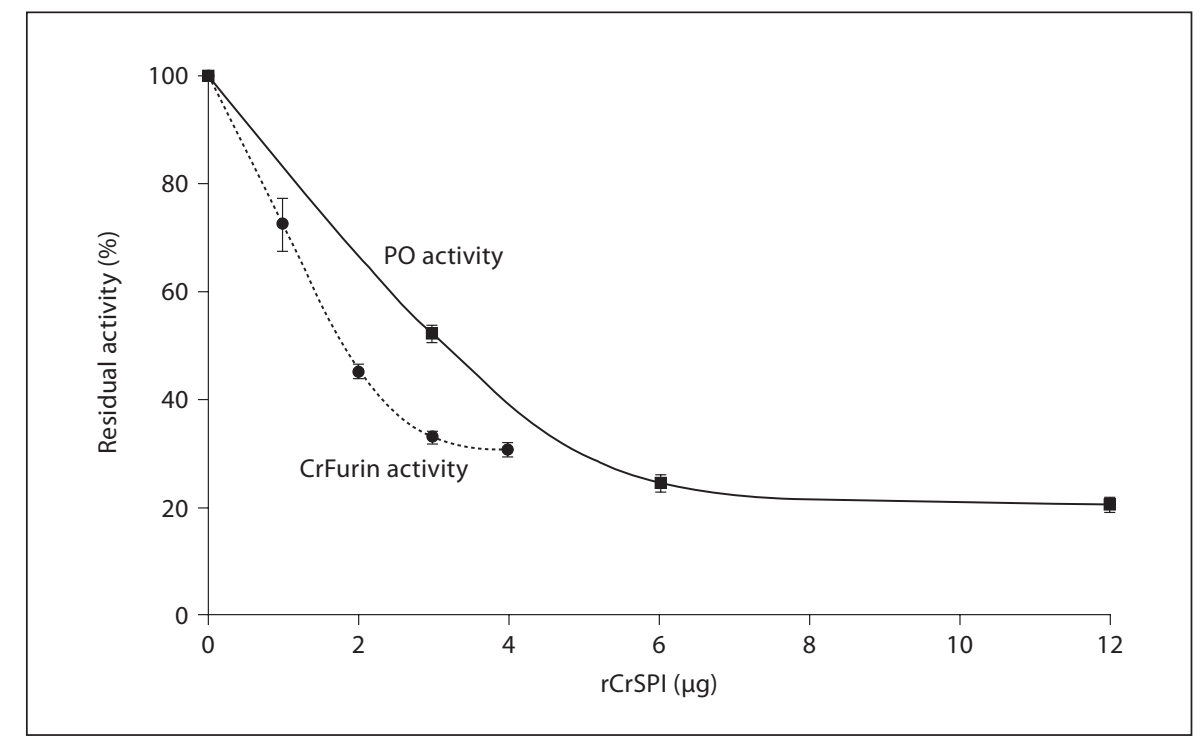

subtilisin A could convert horseshoe crab hemocyanin from PPO to PO with antimicrobial consequences [7]. The activity of CrSPI to inhibit subtilisin-induced PPO activation was measured using purified horseshoe crab hemocyanin and rCrSPI. As shown in figure 7 (solid line), our results demonstrated that the $\mathrm{PO}$ activity induced by subtilisin was downregulated by CrSPI in a dose-dependent manner. In particular, $12 \mu \mathrm{g}$ rCrSPI was able to inhibit approximately $80 \%$ of the subtilisin-mediated activation of PPO to PO.

\section{CrSPI Interacts with a Complement Component C3}

In order to identify the direct interaction partner of CrSPI from the host, GST pull down was performed using GST-CrSPI fusion protein as bait. The plasma and other tissue lysates were examined. As a positive control, subtilisin $\mathrm{A}$, which is the cognate protease of CrSPI, was pulled down (online suppl. fig. 3), and confirmed by MSMS analysis, demonstrating that this GST pull down protocol had substantial sensitivity and specificity. The pull down profile is shown in figure 8a. Bands which occurred only in the GST-CrSPI bait lane were further identified by MS analysis. The $52-\mathrm{kDa}$ protein from the plasma was identified as the p50 component of the C. rotundicauda complement C3, $\mathrm{CrC} 3$ [48], with a detected peptide coverage of $18.6 \%$ of the full length amino acid sequence (fig. 8b, highlighted in bold). As shown in figure 8a, p52 was only isolated from the plasma but not other tissues. Meanwhile, MS-MS of other bands did not reveal interpretable protein identity. The p50 fragment of $\mathrm{CrC} 3$ corresponds to the $\mathrm{C} 3 \mathrm{dg}$ fragment of human $\mathrm{C} 3$, which is a serine protease itself and contains the thioester group required for microbial opsonization. The identification of $\mathrm{C} 3 / \mathrm{C} 3 \mathrm{dg}$ as another of the host's cognate plasma proteases of CrSPI besides CrFurin, suggests that the CrSPI is secreted into the plasma where it performs its multiple potential functions. In view of its interaction with C3, it is conceivable that CrSPI only exists transiently in the plasma, prior to its clearance together with the pathogen via phagocytosis by the hemocytes. Hemocyte-mediated phagocytosis has been demonstrated to be a very efficient process [48].

\section{Discussion}

In this study, we have identified a novel 2-domain Kazal-like SPI from the horseshoe crab, referred to as CrSPI. To reveal the mechanism underlying how a dynamic immune modulation is achieved in a timely manner during the acute phase of infection, we examined the temporal kinetics of CrSPI and its cognate protease, CrFurin, upon $P$. aeruginosa infection. $P$. aeruginosa is present abundantly in the natural muddy-swamp habitat of this species of horseshoe crab, and our earlier studies $[2,7,23,48]$ have shown that $P$. aeruginosa is a pathogen of the horseshoe crab. Therefore it was pertinent to infect this host with $P$. aeruginosa in order to mimic the natural infection conditions. A reciprocal transcriptional profile was observed for CrSPI and CrFurin (fig 1a), which appears to correlate with that of the corresponding plasma protein activities (fig. 1b). Furins are serine proteases involved in 


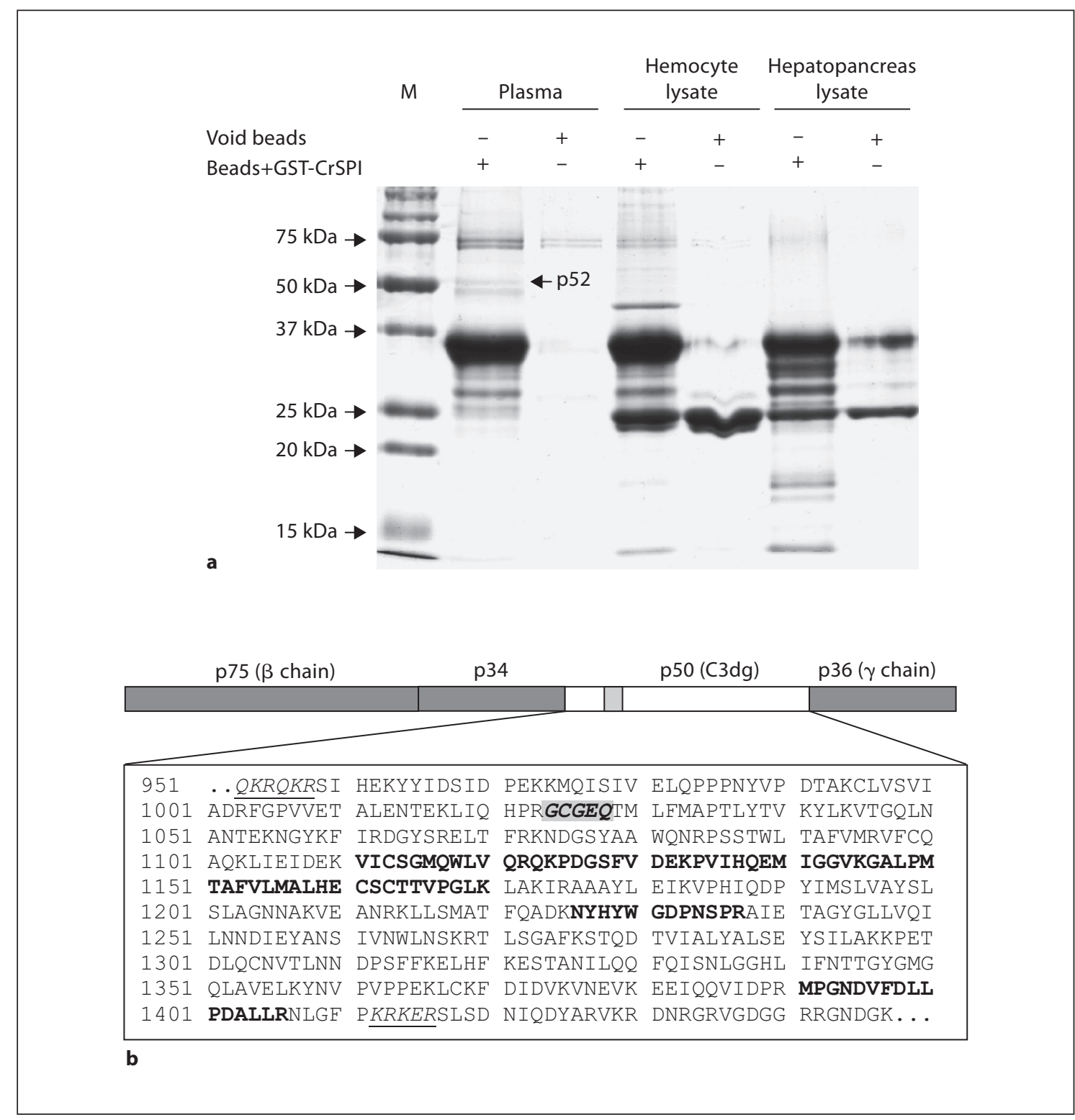

Fig. 8. Identification of $\mathrm{CrC} 3 \mathrm{p} 50$ as one of the cognate proteases of CrSPI in the horseshoe crab plasma. a GST pull down of CrSPI using the hemocyte and hepatopancreas lysates. The pulled down proteins were resolved on $12 \%$ SDS PAGE. The $52-\mathrm{kDa}$ band (arrow) was identified by mass spectrometry as the p50 component

converting immunoproproteins into mature active proteins [20-22]. Therefore the reciprocal dynamics of CrSPI and CrFurin strongly suggest that the host employs $\mathrm{CrSPI}$ as a real-time modulator of serine protease-driven immune responses. It is conceivable that the downregulation of CrSPI transcription during the first hour of infection would limit the level of CrSPI protein and thus allows the protease-driven immune responses to occur; whereas of CrC3. $\mathbf{b}$ The amino acid sequence of p50 (C3dg) component of $\mathrm{CrC} 3$. Peptides detected by mass spectrometry are in bold. The cleavage sites of p50 are underlined and the reactive thioester site is highlighted in grey.

subsequently after 3 h.p.i., restoration of CrSPI transcription 'switches off' such protease-driven immune responses. Such a tightly modulated immune response network is corroborated by our observation that the plasma CrFurin protease activity was elevated at 1 h.p.i. but suppressed to a minimum at 3 h.p.i.

Our attempt to demonstrate the tissue distribution of CrSPI by immunoblot analysis showed no mature CrSPI 
of $9.4 \mathrm{kDa}$ (fig. $2 \mathrm{~b}$ ) in the plasma, but only the $12-\mathrm{kDa}$ band in the hepatopancreas lysate. This observation was apparently incongruent to our expectation since (1) CrSPI is led by a typical secretory signal (fig. 3a) and (2) the CrSPI interacting partners are present in the plasma (fig. $1 \mathrm{~b}$ and $8 \mathrm{a}$ ). However, the Northern blot showed CrSPI to be exclusively expressed in the hepatopancreas (fig. 2a). We believe that within the tissues, the hepatopancreas would harbor the nascent protein precursor of $12 \mathrm{kDa}$ (fig. 2b). After processing and secretion, the mature active form of 9.4-kDa CrSPI probably exists transiently in the plasma (detectable by more sensitive biochemical activity assay; fig. 1b). This is especially so during innate immune defense where the CrSPI performs its function by forming a complex with its cognate protease which undergoes phagocytosis by the hemocytes [48]. This is corroborated by its lowest activity during 1-3 h.p.i. (fig. 1b).

Surprisingly, we observed a CrSPI-containing protein band of $>100 \mathrm{kDa}$ in the hemocyte lysate and in the hemocyanin-free plasma spiked with the 5-kDa rCrSPI-D2 protein (fig. 2b). As shown in figure $2 \mathrm{~b}$, the Western blotting protocol could readily detect $10 \mathrm{ng}$ of the recombinant CrSPI-D2 protein, thus confirming the validity of these protein profiles. Taken together, our observations raised 2 main questions: what is the basis for the observed CrSPI profile, and what is the biological significance of CrSPI to the host's antimicrobial response? The remaining discussion attempts to provide answers to these questions using recombinant CrSPI.

To demonstrate the inhibitory characteristics of this Kazal-like SPI, we isolated cDNA clones of CrSPI and further subcloned its domains. The atypical lengths of the CrSPI domains, the unusual Cys residue distribution, and the unique molecular conformations all warrant its classification amongst nonclassical Kazal inhibitors. Amongst various serine proteases tested, we found that subtilisin was the most susceptible and most strongly inhibited by rCrSPI. Additionally, our preliminary experiments using $P$. aeruginosa cell lysate demonstrated that rCrSPI substantially inhibited the subtilisin-like proteases from $P$. aeruginosa (online suppl. fig. 4). Therefore, we used subtilisin A as a model serine protease to address the inhibitory characteristics of CrSPI against a microbial protease, including the elucidation of its stoichiometry and Ki value. We found that rCrSPI shows high inhibitory activity against subtilisin, at a $\mathrm{Ki}$ of $2.76 \mathrm{nM}$ (fig. 5). Furthermore, the 1:2 stoichiometry of complex formation between rCrSPI and subtilisin A (fig. 6) seemed to suggest that both domains of the CrSPI are function- ally active and each domain by itself could bind 1 subtilisin A molecule. This is further supported by the 1:1 stoichiometry between rCrSPI-D2 and subtilisin A. However, the lack of inhibitory activity by rCrSPI-D1 alone suggests that the intact rCrSPI is essential for a fully functional CrSPI, which infers that D1 may be an autoregulatory domain, although further studies will be required to confirm this.

Although subtilisin A is apparently better associated with $B$. subtilis, subtilisin homologs have been revealed to be virulence factors and they play important roles in the pathogenesis of Pseudomonas infection. These subtilisin homologs include, for example, protease IV, an extracellular serine protease with a molecular mass of approximately $26 \mathrm{kDa}$, and AprA, an alkaline subtilisin-like protease [21, 36-38]. To reveal a universal/common strategy that the host organism may exploit to combat the invading microbes, it would have been ideal for us to test the inhibitory characteristics of CrSPI to these Pseudomonas proteases. However, the lack of such commercially available enzymes hindered us from conducting such a test. Nevertheless, our preliminary experiments using $P$. $a e$ ruginosa cell lysate have demonstrated that rCrSPI significantly inhibits the subtilisin-like proteases from $P$. aeruginosa (online suppl. fig. 4).

To fully appreciate the function of CrSPI, it was essential to identify its native cognate protease under physiological conditions as well as during infection. rCrSPIGST pull down assay showed that during infection, this serine protease inhibitor interacts with microbial subtilisin as well as the host endogenous C3 complement component, p50 (fig. 8). Dose-dependent inhibitory activity assay revealed CrFurin as another possible host protease whose activity is also regulated by CrSPI (fig. 7). All 3 proteins regulated by CrSPI play important roles in homeostasis and immune response. The first, the p50 fragment of $\mathrm{CrC} 3$, corresponds to the $\mathrm{C} 3 \mathrm{dg}$ fragment of human C3, which contains the thioester group required for microbial/particle opsonization (fig. 8). The second protein, Furin, which is a subtilisin homolog, is involved in converting immunoproproteins into mature active proteins [20-22]. The third, subtilisin, although of microbial origin, has recently been demonstrated [7] to be exploited by the host to initiate a highly efficient antimicrobial strategy through activating host PO. The important roles of its cognate proteases have justified CrSPI as a multitargeting immunomodulator.

The unique biochemical property of CrSPI's cognate proteases, which are the complement protease and microbial protease subtilisin A, prompted a plausible explana- 
tion as follows. Once secreted into plasma, CrSPI is swiftly consumed by its cognate protease through complex formation, and is promptly scavenged by hemocyte-mediated phagocytosis. Such a hemocyte-mediated phagocytosis event has been previously observed in the horseshoe crab antimicrobial immune response [48]. Along this line of reasoning, we postulate that the $>100-\mathrm{kDa}$ CrSPI-containing protein in the hemocyte lysate (fig. $2 \mathrm{~b}$, lanes 4-6) could be the complex formed between CrSPI and its cognate protease which was thereafter phagocytosed by the hemocyte; whereas the $>100-\mathrm{kDa}$ band in the hemocyanin-free plasma spiked with $5-\mathrm{kDa}$ rCrSPID2 (fig. 2b, lane 9) could represent a complex formed between CrSPI-D2 and its cognate protease in the plasma. Furthermore, the very high inhibitory constant (Ki of $10^{-9} \mathrm{M}$ ) of CrSPI for its cognate protease (fig. 5) would have ensured their expeditious partnership with consequential clearance of the CrSPI:serine protease complex.

In vivo, there are multiple serine protease-driven immune response pathways [8]. As a proof of concept, we have demonstrated that subtilisin-induced PPO activation is readily downregulated by $\mathrm{rCrSPI}$ (fig. 7, solid line).
In future studies, in order to confirm a universal/common strategy that the host may exploit against both Gram-positive and Gram-negative bacteria, the in vivo infection by Gram-positive bacteria such as B. subtilis may be included to analyze the effects on the CrSPI.

In summary, we have demonstrated that as a multitargeting immunomodulator, CrSPI regulates serine protease-driven antimicrobial responses at the acute phase (16 h.p.i.) of host immune defense while maintaining homeostasis under naïve condition. The versatility of SPI contributes significantly to maintaining an intricate immunomodulatory balance in frontline innate immune defense.

\section{Acknowledgements}

We thank the following funding agencies for financing this research: Agency for Science Technology and Research $\left(\mathrm{A}^{*} \mathrm{STAR}\right.$ BMRC grant); Ministry of Education (AcRF Tier 2 grant to J.L.D., B.H. and J.S.); and the Faculty of Science Research Fund, National University of Singapore (FRC grant to J.L.D.).

\section{References}

1 Ding JL, Navas MAA, Ho B: Two forms of factor C from the amoebocytes of Carcinoscorpius rotundicauda: Purification and characterization. Biochim Biophys Acta 1993;1202:149-156.

- 2 Ding JL, Tan K, Thangamani S, Kusuma N, Seow WK, Bui THH, Wang J, Ho B: Spatial and temporal coordination of expression of immune response genes during Pseudomonas infection of horseshoe crab, Carcinoscorpius rotundicauda. Genes Immun 2005;6: 557-574.

3 Iwanaga S, Miyata T, Tokunaga F, Muta T: Molecular mechanism of hemolymph clotting system in limulus. Thromb Res 1992;68: $1-32$.

4 Rosenmund A, Haeberli A, Straub PW: Blood coagulation and acute iron toxicity. Reversible iron-induced inactivation of serine proteases in vitro. J Lab Clin Med 1984;103:524533.

5 Michel T, Reichhart J-M, Hoffmann JA, Royet J: Drosophila toll is activated by gram-positive bacteria through a circulating peptidoglycan recognition protein. Nature 2001;414: 756-759.

6 Cerenius L, Soderhall K: The prophenoloxidase-activating system in invertebrates. Immunol Rev 2004;198:116-126.
7 Jiang N, Tan NS, Ho B, Ding JL: Respiratory protein-generated reactive oxygen species as an antimicrobial strategy. Nat Immunol 2007;8:1114-1122.

-8 Le Saux A, Ng PML, Koh JJY, Low DHP, Leong GEL, Ho B, Ding JL: The macromolecular assembly of pathogen-recognition receptors is impelled by serine proteases, via their complement control protein modules. J Mol Biol 2008;377:902-913.

-9 Hiemstra PS: Novel roles of protease inhibitors in infection and inflammation. Biochem Soc Trans 2002;30:116-120.

10 Armstrong PB: The contribution of proteinase inhibitors to immune defense. Trends Immunol 2001;22:47-52.

11 Chavanas S, Bodemer C, Rochat A, HamelTeillac D, Ali M, Irvine AD, Bonafe J-L, Wilkinson J, Taieb A, Barrandon Y, Harper JI, de Prost Y, Hovnanian A: Mutations in SPINK5, encoding a serine protease inhibitor, cause Netherton syndrome. Nat Genet 2000;25:141-142.

12 Iwanaga S: The molecular basis of innate immunity in the horseshoe crab. Curr Opin Immunol 2002;14:87-95.

13 Laskowski M, Kato I: Protein inhibitors of proteinases. Ann Rev Biochem 1980;49:593626.
14 Bode W, Huber R: Natural protein proteinase inhibitors and their interaction with proteinases. Eur J Biochem 1992;204:433-451.

15 Somprasong N, Rimphanitchayakit V, Tassanakajon A: A five-domain Kazal-type serine proteinase inhibitor from black tiger shrimp Penaeus monodon and its inhibitory activities. Dev Comp Immunol 2006;30: 998-1008.

16 Nirmala X, Kodrik D, Zurovec M, Sehnal F: Insect silk contains both a Kunitz-type and a unique Kazal-type proteinase inhibitor. Eur J Biochem 2001;268:2064-2073.

17 Gebhard LG, Carrizo FU, Stern AL, Burgardt NI, Faivovich J, Lavilla E, Ermacora MR: A Kazal prolyl endopeptidase inhibitor isolated from the skin of Phyllomedusa sauvagii. Eur J Biochem 2004;271:2117-2126.

18 Kumazaki T, Ishii S-I: Disulfide bridge structure of ascidian trypsin inhibitor I: Similarity to Kazal-type inhibitors. J Biochem 1990;107:414-419.

19 Johansson MW, Keyser P, Soderhall K: Purification and cDNA cloning of a four-domain Kazal proteinase inhibitor from crayfish blood cells. Eur J Biochem 1994;223:389394.

20 Nakayama K: Furin: a mammalian subtili$\sin /$ Kex2p-like endoprotease involved in processing of a wide variety of precursor proteins. Biochem J 1997;327:625-635. 
21 Rawlings ND, Tolle DP, Barrett AJ: Merops: the peptidase database. Nucleic Acids Res 2004;32:D160-D164

22 Rockwell NC, Krysan DJ, Komiyama T, Fuller RS: Precursor processing by Kex $2 /$ Furin proteases. Chem Rev 2002;102:4525-4548.

-23 Ng PML, Zhenxiao J, Tan SSH, Ho B, Ding JL: C-reactive protein: a predominant LPSbinding acute phase protein responsive to Pseudomonas infection. J Endotoxin Res 2004;10:163-174.

24 Donnes P, Elofsson A: Prediction of MHC class I binding peptides, using SVMHC. BMC Bioinformatics 2002;3:25.

25 Lamango NS, Zhu X, Lindberg I: Purification and enzymatic characterization of recombinant prohormone convertase 2: stabilization of activity by $21 \mathrm{kDa} 7 \mathrm{~b} 2$. Arch Biochem Biophys 1996;330:238-250.

-26 Bendtsen JD, Nielsen H, Heijne Gv, Brunak S: Improved prediction of signal peptides: Signalp 3.0. J Mol Biol 2004;340:783-795.

27 Soding J: Protein homology detection by HMM-HMM comparison. Bioinformatics 2005;21:951-960.

28 Soding J, Biegert A, Lupas AN: The HHpred interactive server for protein homology detection and structure prediction. Nucleic Acids Res 2005;33:W244-W248.

-29 Canutescu AA, Shelenkov AA, Dunbrack RL Jr: A graph-theory algorithm for rapid protein side-chain prediction. Protein Sci 2003; 12:2001-2014.

- 30 Altschul S, Gish W, Miller W, Myers W, Lipman DJ: Basic local alignment search tool. J Mol Biol 1990;215:403-410.

- 31 Kopacek P, Weise C, Saravanan T, Vitova K, Grubhoffer L: Characterization of an alphamacroglobulin-like glycoprotein isolated from the plasma of the soft tick Ornithodoros moubata. Eur J Biochem 2000;267:465-475.
32 Jayakumar A, Kang Ya, Mitsudo K, Henderson Y, Frederick MJ, Wang M, El-Naggar AK, Marx UC, Briggs K, Clayman GL: Expression of LEKTI domains 6-9' in the baculovirus expression system: Recombinant LEKTI domains 6-9' inhibit trypsin and subtilisin A. Protein Expr Purif 2004;35:93101.

33 Mitsudo K, Jayakumar A, Henderson Y, Frederick MJ, Kang Y, Wang M, El-Naggar AK, Clayman GL: Inhibition of serine proteinases plasmin, trypsin, subtilisin A, cathepsin G, and elastase by LEKTI: a kinetic analysis. Biochemistry 2003;42:3874-3881.

34 Morris MT, Coppin A, Tomavo S, Carruthers VB: Functional analysis of Toxoplasma gondii protease inhibitor 1. J Biol Chem 2002; 277:45259-45266.

-35 Lingaraju MH, Gowda LR: A Kunitz trypsin inhibitor of Entada scandens seeds: another member with single disulfide bridge. Biochim Biophys Acta 2008;1784:850-855

36 Caballero AR, Moreau JM, Engel LS, Marquart ME, Hill JM, O'Callaghan RJ: Pseudomonas aeruginosa protease IV enzyme assays and comparison to other Pseudomonas proteases. Anal Biochem 2001;290:330-337.

37 Engel LS, Hill JM, Caballero AR, Green LC, O'Callaghan RJ: Protease IV, a unique extracellular protease and virulence factor from Pseudomonas aeruginosa. J Biol Chem 1998; 273:16792-16797.

- 38 Traidej M, Marquart ME, Caballero AR, Thibodeaux BA, O'Callaghan RJ: Identification of the active site residues of Pseudomonas aeruginosa protease IV. Importance of enzyme activity in autoprocessing and activation. J Biol Chem 2003;278:2549-2553.

39 Mende K, Petoukhova O, Koulitchkova V, Schaub GA, Lange U, Kaufmann R, Nowak G: Dipetalogastin, a potent thrombin inhibitor from the blood-sucking insect Dipetalogaster maximus. cDNA cloning, expression and characterization. Eur J Biochem 1999; 266:583-590.
40 Kellenberger C, Boudier C, Bermudez I, Bieth JG, Luu B, Hietter H: Serine protease inhibition by insect peptides containing a cysteine knot and a triple-stranded betasheet. J Biol Chem 1995;270:25514-25519.

41 Bode W, Huber R: Proteinase-protein inhibitor interaction. Biomed Biochim Acta 1991; 50:437-446.

42 Barrette-Ng IH, Ng KK-S, Cherney MM, Pearce G, Ryan CA, James MNG: Structural basis of inhibition revealed by a 1:2 complex of the two-headed tomato inhibitor-ii and subtilisin Carlsberg. J Biol Chem 2003;278: 24062-24071.

43 Magert H-J, Standker L, Kreutzmann P, Zucht H-D, Reinecke M, Sommerhoff CP, Fritz H, Forssmann W-G: LEKTI, a novel 15domain type of human serine proteinase inhibitor. J Biol Chem 1999;274:21499-21502.

44 Hatsuzawa K, Nagahama M, Takahashi S Takada K, Murakami K, Nakayama K: Purification and characterization of Furin, a Kex2-like processing endoprotease, produced in Chinese hamster ovary cells. J Biol Chem 1992;267:16094-16099.

45 Vidricaire G, Denault JB, Leduc R: Characterization of a secreted form of human Furin endoprotease. Biochem Biophys Res Commun 1993;195:1011-1018.

46 Vey M, Schafer W, Berghofer S, Klenk H, Garten W: Maturation of the trans-Golgi network protease furin: compartmentalization of propeptide removal, substrate cleavage, and COOH-terminal truncation. J Cell Biol 1994;127:1829-1842.

47 Thimon V, Belghazi M, Dacheux J-L, Gatti J-L: Analysis of furin ectodomain shedding in epididymal fluid of mammals: demonstration that shedding of furin occurs in vivo. Reproduction 2006;132:899-908.

48 Zhu Y, Thangamani S, Ho B, Ding JL: The ancient origin of the complement system. EMBO J 2005;24:382-394. 